\title{
Prevalence and severity of symptoms and signs in patients with advanced cancer in the last days of life: the East Asian collaborative cross-cultural Study to Elucidate the Dying process (EASED)
}

Yusuke Hiratsuka ( $\nabla$ hiratsuka.med.t@gmail.com )

Tohoku Daigaku https://orcid.org/0000-0003-2334-3893

Sang-Yeon Suh

Dongguk University

Seon-Hye Won

Dongguk University

Sun-Hyun Kim

Catholic Kwandong University

Seok-Joon Yoon

Chungnam National University

Su-Jin Koh

Ulsan University Hospital

Jung Hye Kwon

Chungnam National University

Jeanno Park

Bobath Memorial Hospital

Hong-Yup Ahn

Dongguk University

Shao-Yi Cheng

National Taiwan University

Ping-Jen Chen

Kaohsiung Medical University

Takashi Yamaguchi

Kobe University: Kobe Daigaku

Tatsuya Morita

Seirei Mikatahara Byoin

Satoru Tsuneto

Kyoto Daigaku

Masanori Mori

Seirei Mikatahara Byoin 


\section{Akira Inoue}

Tohoku University: Tohoku Daigaku

\section{Research Article}

Keywords: Palliative care, Advanced cancer, End of life, Signs and symptoms, East Asia

Posted Date: November 4th, 2021

DOl: https://doi.org/10.21203/rs.3.rs-881698/v1

License: (c) (1) This work is licensed under a Creative Commons Attribution 4.0 International License. Read Full License

Version of Record: A version of this preprint was published at Supportive Care in Cancer on March 19th, 2022. See the published version at https://doi.org/10.1007/s00520-022-06969-9. 


\section{Abstract}

Purpose Few large-scale studies have focused on the prevalence of symptoms and signs during the last days of patients diagnosed with advanced cancer. Identifying the patterns of specific symptoms according to cancer type is helpful to provide end-of-life care for patients with advanced cancer. We investigated the prevalence and severity of symptoms and signs associated with impending death in patients with advanced cancer. Methods In this secondary analysis of an international multicenter cohort study conducted in three East Asian countries, we compared the severity of symptoms and signs among dying patients 3 days before death according to the type of primary cancer using one-way analysis of variance (ANOVA). Post hoc analysis was conducted for multiple comparisons of each symptom according to the type of primary cancer. Results We analyzed 2131 patients from Japan, Korea and Taiwan. Fatigue, dry mouth, drowsiness and dyspnea were present in nearly half of the patients, and edema of lower extremities was the most common sign in all dying patients. According to cancer type, edema of lower extremities was the most common symptom and fatigue/ ascites were the most severe symptoms in digestive tract cancer. For lung cancer, respiratory secretion was the most prevalent and dyspnea/ respiratory secretion were the most severe symptoms. Conclusion We demonstrated the prevalence and severity of symptoms and signs associated with impending death of patients with advanced cancer in East Asia. Our study can enable clinicians to recognize the specific symptoms and signs at the very end of life.

\section{Introduction}

Patients experience a variety of physical and psychological symptoms as they approach the last days of their lives [1]. A better understanding of various symptoms associated with impending death can enable clinicians improve the diagnostic performance and palliative patients [2]. However, few studies have focused on the prevalence of symptoms during the last days of patients on a large scale [1, 3-8]. These studies focused on the specific symptoms recognized in actively dying patients. Therefore, the frequency of common clinical symptoms such as fatigue and dyspnea in the last days has yet to be systematically analyzed. In a systematic review that included both cancer patients and non-cancer patients, dyspnea, pain, respiratory secretions/death rattle and confusion were the most prevalent symptoms in the last 2 weeks of life [2]. A recent study involving East Asian countries has reported the prevalence of leg edema and ascites in patients with pancreaticobiliary cancer and myoclonus and respiratory secretions in patients with gastroesophageal cancer 3 days before death [9].

Identification of the pattern of specific symptoms and signs according to cancer type facilitates our understanding of the characteristics of patients with specific cancer type and provide end-of-life care. Thus, we investigated the prevalence and severity of symptoms and signs associated with impending death in patients with advanced cancer in three East Asian countries.

\section{Methods}




\section{Study participants}

This is a secondary analysis of an international multicenter cohort study conducted in three East Asian countries including Japan, Korea and Taiwan. The original study was the East Asian collaborative crosscultural Study to Elucidate the Dying Process (EASED), which investigated the dying process and end-oflife care of inpatients with far advanced cancer in palliative care units (PCUs) in three East Asian countries. Eligible, newly admitted inpatients in the participating PCUs during the study period were consecutively enrolled in the study. All observations were performed in the course of routine clinical practice. The inclusion criteria were as follows: (1) adults (age $>=18$ years in Japan and Korea and $>=$ 20 years in Taiwan), (2) suffering from locally extensive or metastatic cancer, and (3) admitted to a participating PCU. The exclusion criteria were: (1) scheduled discharge within 1 week and (2) refusal by patients or their families to participate in the study.

\section{Data collection}

The attending palliative care physicians and nurses recorded all demographic data on admission, and symptoms in the last 3 days of life based on the structured questionnaire. The questionnaire was developed through discussions among EASED investigators and the results of both western and eastern studies analyzing "impending death" and "good communication practices" involving dying patients and their families in PCUs [10-15].

We obtained and analyzed the following data: patient demographics and clinical characteristics on admission, and symptoms and signs in the last 3 days of life. Symptoms were defined as observations of patients because subjective assessment was difficult in the last 3 days of life. Symptoms included fatigue, dry mouth, drowsiness, dyspnea, delirium and hallucination. We used the Integrated Palliative Outcome Scale (IPOS) to objectively measure patients' symptoms including fatigue, dry mouth and drowsiness: 0 , not at all; 1 , slightly; 2 , moderately; 3 , severely; 4 , overwhelmingly; 5 , cannot assess because of unconsciousness. The Japanese version of IPOS is a valid and reliable tool for assessing physical, psychological, social, and spiritual symptoms and measuring the outcomes of adult cancer patients in Japan [16]. The IPOS is currently undergoing validation in Korea. Taiwanese investigators have completed the validation of the IPOS and reported the results at an international symposium (Tohoku Forum for Creativity 2020). Dyspnea was scored as follows: 0, normal; 1, exertional only; and 2, at rest. We assessed delirium using the 10-item Memorial Delirium Assessment Scale (MDAS), based on item \# 9 (psychomotor activity): 0, normal or psychomotor hypoactivity; 1 , mild; 2 , moderate; and 3 , severe [17]. Hallucination was evaluated using the Delirium Rating Scale-revised-98 (DRS-R-98), with respect to item \# 2 (perceptual disturbances and hallucinations): 0, not present; 1, mild perceptual disturbance; 2 , illusions present; and 3, hallucinations present [18]. Respiratory secretion was scored as: 0 , not audible; 1 , only audible at the head of the bed; 2 , clearly audible at the foot of the bed; and 3 , clearly audible $6 \mathrm{~m}$ away from the foot of the bed [19]. The severity of leg edema was determined by measuring the depth upon pressing the skin for a sufficient amount of time in the area between the upper and the lower joints and was categorized as follows: 0 , none; 1 , mild ( $<5 \mathrm{~mm}) ; 2$, moderate $(5-10 \mathrm{~mm})$; and 3 , 
severe (> $10 \mathrm{~mm})[20,21]$. Ascites was scored as follows: 0 , physically undetectable; 1 , physically detectable but asymptomatic; or 2 , symptomatic based on physician examination. We evaluated myoclonus based on five levels of jerking frequency per $10 \mathrm{~s}$ at rest: 0 , none; $1, \leq 1$ jerk; $2,2-3$ jerks; 3, 49 jerks; and $4, \geq 10$ jerks.

\section{Statistical analysis}

All analyses were performed using JMP version 14 for Windows (SAS, Cary, NC, USA).

First, we classified study participants into 3 groups according to the type of primary cancer prevailing in East Asia: digestive tract, lung and others. Digestive tract cancer includes gastroesophageal, colorectal, hepatobiliary, and pancreatic cancer. Lung cancer includes small-cell and non-small cell types. Other cancers include breast, gynecological, urological, head and neck, bone and soft tissue, skin, brain cancer and hematological malignancies.

Second, we performed descriptive analyses to summarize baseline characteristics of study participants according to the type of primary cancer.

Third, descriptive analyses were summarized as the prevalence of physical and psychological symptoms and signs according to the type of primary cancer in patients at admission, 1 week after admission and in the last 3 days of life. We defined 'present symptoms' as having more than one point of score of the response scale.

Fourth, we compared the severity of physical and psychological symptoms and signs in the last 3 days of life according to the type of primary cancer using one-way analysis of variance (ANOVA). Post hoc analysis was conducted using Tukey-Kramer test for multiple comparisons of each symptom according to the type of primary cancer.

\section{Ethics}

In accordance with the ethical guidelines for human research of Japan's Ministry of Health, Labor, and Welfare, informed patient consent was waived in Japan because of the completely observational nature of the study. In Korea and Taiwan, informed consent was obtained from the patients or their families (if the patient lacked the capacity to make decisions). The study was approved by the local Institutional Review Boards of all participating institutions. Also, the independent ethics committee of Tohoku University School of Medicine (approval no. 2016-1-689) approved this study.

\section{Results}

\section{Patient characteristics}

A total of 2638 patients were enrolled across 38 palliative care units (23 in Japan, 11 in South Korea, and 4 in Taiwan). The patients were enrolled from January 2017 to September 2018. We excluded 500 
ineligible patients (digestive tract: 213 , lung: 72 , others: 215 ) because they were discharged alive. Among 2138 eligible patients, 7 patients (digestive tract: 2, lung: 5, others: 0) were excluded for missing values of all symptoms in the last 3 days of life. Thus, a total of 2131 patients (digestive tract: 1042, lung: 368, others: 721) were evaluated (Figure 1) including 1107 men (digestive tract: 575 (55.2\%), lung: 239 (65.0\%), others: $293(52.6 \%)$ ). Japanese patients who participated in the study were as follows: $74.6 \%$, digestive tract group; $76.9 \%$, lung group; $79.2 \%$, others. The median survival time was 15 days $(95 \%$ confidence interval [Cl], 2.0-71.0) in the digestive tract group, 15 days $(95 \% \mathrm{Cl}, 2.0-90.2)$ in lung group and 15 days $(95 \% \mathrm{Cl}, 2.0-79.0)$ in the others group. The characteristics of the patients are listed in Table 1 .

\section{Prevalence of symptoms and signs}

The prevalence of physical and psychological symptoms and signs according to the type of primary cancer in patients in the last 3 days of life is presented in Table 2. The changes in the prevalence of symptoms and signs are shown in Figure 2. Fatigue, dry mouth, drowsiness and dyspnea were the most frequent symptoms detected in nearly half of all patients (41.1-56.9\%), and edema of lower extremities was the most common sign (62.4\%). For digestive tract cancer, edema of lower extremities was the most common sign (68.1\%) in the last 3 days of life. Respiratory secretion was the most prevalent sign (56.1\%) associated with lung cancer in the last 3 days of life. As for other types of cancer, edema of lower extremities was the most predominant sign (62.6\%) in the last 3 days of life. The proportion of unconsciousness was up to $35.8-36.8 \%$.

Severity of symptoms and signs

The severity of physical and psychological symptoms and signs in the last 3 days of life according to the type of primary cancer is presented in Table 3 . The severities of six symptoms and signs varied significantly according to the type of primary cancer after ANOVA tests: fatigue $(p=0.01)$, dry mouth ( $p=$ $0.03)$, dyspnea $(p<0.01)$, respiratory secretion $(p<0.01)$, edema of lower extremities $(p<0.01)$ and ascites $(p<0.01)$. Post-hoc analysis revealed that fatigue and ascites were significantly more severe in patients with digestive tract cancer, while dyspnea and respiratory secretion were significantly more severe in lung cancer.

\section{Discussion}

The aim of this study was to investigate the prevalence and severity of symptoms and signs associated with impending death in patients with advanced cancer in three East Asian countries. Fatigue, dry mouth, drowsiness and dyspnea were present in nearly half of patients, and edema of lower extremities was the most common sign in all dying patients. According to cancer type, edema of lower extremities was the most common symptom and fatigue/ascites were the most severe symptoms in digestive tract cancer. For lung cancer, respiratory secretion was the most prevalent and dyspnea/ respiratory secretion were the most severe symptoms. 
Fatigue, drowsiness and dry mouth were reported in nearly half of dying patients in our study. Fatigue and dry mouth can be regarded as manifestations of the cancer-anorexia-cachexia syndrome, which was frequent in patients with advanced cancer [22, 23]. Drowsiness is also one of the poor prognostic factors associated with survival and may be interpreted as decreased level of consciousness in dying patients $[24,25]$. Thus it is no wonder that such symptoms were most common in the last 3 days of life. Dyspnea is one of the key symptoms with the highest prevalence in the last days of life. According to a systematic review, the increased prevalence of dyspnea was $62.1 \%$ [26]. Our results showed a $41.1 \%$ prevalence of dyspnea, which was slightly lower than in those reported in the systematic review. Since our patients were inpatients of PCUs, palliative care may alleviate dyspnea. Notably, edema of lower extremities was the most common sign (62.4\%) of impending death in the current study. Edema is a component of palliative prognostic index, which is a valid prognostic score worldwide [27]. The index was used to predict 3-week survival in our population.

Edema of lower extremities was the most prevalent symptom associated with digestive tract cancer, and ascites was the more severe sign. Frequent hepatic metastases in patients result in edema of lower extremities and ascites [28]. Based on the severity of symptoms, fatigue was the most severe symptom in patients with digestive tract cancer than in any other types of cancer. In a Canadian study [29], similar to ours, fatigue was the most severe symptom in patients with gastrointestinal cancer.

Respiratory secretion was the most frequent and severe sign in lung cancer. Previous studies [30, 31] reported lung cancer as the underlying etiology of "death rattle". In addition, the higher prevalence of brain metastasis in lung cancer may be related to frequent respiratory secretion [30, 32]. It is well known that lung cancer is associated with pneumonia including obstructive pneumonia [33], which is related to the high frequency of death rattle [31]. We assumed several factors may reinforce the intensity of the respiratory secretion. Dyspnea was most severe symptom in lung cancer than in other types of cancer. The intensity of dyspnea in any cancer type is moderate to severe [34, 35], and higher in primary and metastatic lung cancer $[36,37]$. These figures are the highest at the end of life $[38,39]$ and consistent with our findings.

The strengths of our study are as follows. This was the first large-scale study reporting the prevalence and severity of symptoms and signs associated with impending death in patients with advanced cancer in East Asian countries. Our findings reflect the cross-cultural demands in the last days of life. For instance, the recognition can be helpful for survival prediction and preparation of medical professionals and family; unmet business of Japanese families [40]. Korean palliative care professionals should decide when to move to a private room before 96 hours of death, which is covered by national health insurance in Korea. Taiwanese regard 'returning to home when death comes very nearer' as an important component of good death [41]. Another strength is that we predefined the assessment of severities and the timing of symptoms and signs based on consensus among the international investigators.

We recognize that this study has several limitations. First, we used IPOS for assessment of symptoms. It was previously reported that the inter-class correlation coefficients (ICCs) of fatigue, drowsiness and dry 
mouth for IPOS-Patient and IPOS-Staff were 0.646 (which meant substantial relationship), 0.593 (moderate) and 0.576 (moderate), respectively, in Japan [42]. In Taiwan, ICCs between patients and staff were 0.611 (substantial) for fatigue, 0.867 (good) for drowsiness and 0.785 (good) for dry mouth. Indeed, it was difficult for most patients to subjectively assess IPOS in the last 3 days of life. Therefore, we regarded IPOS-Staff as an acceptable tool for our study. Second, there was a possible recall bias involved in symptom assessment. We evaluated the symptoms after patients' death and recalled the symptoms in the last 3 days of life. Additionally, we were unable to assess symptoms in the last 3 days of life of 35.8$36.8 \%$ patients because of their unconsciousness. As a result, unconscious patients were excluded from analysis in the current study. There was a possibility that the evaluated patients manifested relatively better function. It has been reported that around $70 \%$ patients were unconscious in the last days of life [43]. Therefore, selection bias might be considered in the current study. Third, this study population may not represent the general population of patients with far advanced cancer; the prevalence and severity in our patients may differ from those of patients at home or in general ward. Therefore, our results may not be applicable to the general population of each country. Fourth, medical treatments such as opioid administration may affect the prevalence and severity of symptoms. Fifth, our findings could be affected by unmeasured covariates such as pain score. We did not include other known signs of impending death, such as dysphagia of liquids or decrease in urine output [44]. Lastly, accessibility to palliative care services and palliative care unit admission in each country may have influenced our findings. Therefore, it is necessary to rate the symptoms in the final days of patients who receive care at home or at a specialty other than palliative care units.

\section{Conclusion}

We demonstrated the prevalence and severity of symptoms associated with impending death of patients with advanced cancer in East Asia. Due to the large scale and regional diversity, our study can enable clinicians to recognize the very end-of-life patterns of specific symptoms and signs. Timely awareness of such patterns will lead to enhanced quality of end-of-life care for patients. Further, it can facilitate survival prediction and preparation of medical professionals, patients and their families. Further studies are needed to investigate the prevalence and severity of symptoms and signs associated with impending death of patients with advanced cancer in other palliative care settings.

\section{Declarations}

Acknowledgments

We appreciate EASED Investigators for their sincere data collection.

We are grateful to Harrisco Encorrection (Seoul, Korea) for proofreading this manuscript for grammar and clarity.

Funding 
This work was supported in part by a Grant-in-Aid from the Japanese Hospice Palliative Care Foundation; Grant Numbers $16 \mathrm{H} 05212$ and 16KT0007.

\section{Conflicts of interest}

The authors declare that there is no conflict of interest.

\section{Data availability}

The data that support the findings of this study are available from the corresponding author, Sang-Yeon Suh, upon reasonable request. All authors agree to provide data to the journal for review if needed.

\section{Code availability}

Not applicable.

\section{Author contributions}

Yusuke Hiratsuka: Conceptualization, Data curation, Formal analysis, Investigation, Methodology, Project administration, Writing - original draft, Writing - review and editing

Sang-Yeon Suh: Conceptualization, Investigation, Methodology, Project implementation, Supervision, Writing - review and editing

Seon-Hye Won: Investigation, Writing - review and editing

Sun-Hyun Kim: Investigation, Writing - review and editing

Seok-Joon Yoon: Investigation, Writing - review and editing

Su-Jin Koh: Investigation, Writing - review and editing

Jung Hye Kwon: Investigation, Writing - review and editing

Jeanno Park: Investigation, Writing - review and editing

Hong-Yup Ahn: Formal analysis, Writing - review and editing

Shao-Yi Cheng: Investigation, Writing - review and editing

Ping-Jen Chen: Investigation, Writing - review and editing

Takashi Yamaguchi: Investigation, Writing - review and editing

Tatsuya Morita: Funding acquisition, Investigation, Writing - review and editing

Satoru Tsuneto: Supervision, Writing - review and editing 
Masanori Mori: Conceptualization, Investigation, Methodology, Project administration, Resources, Supervision, Writing - review and editing

Akira Inoue: Investigation, Supervision, Writing - review and editing

\section{Ethics approval}

This study was approved by the local Institutional Review Boards of all participating institutions. The independent ethics committee of Tohoku University School of Medicine (approval no. 2016-1-689) approved this study.

\section{Consent to participate}

In accordance with the ethical guidelines for human research issued by the Ministry of Health, Labor, and Welfare in Japan, patients' informed consent was waived because of the completely observational nature of the study. Patients were provided with the opportunity to opt out. In Korea and Taiwan, informed consent was obtained from the patients or their families (in case the patient lacked the capacity to decide).

\section{Consent for publication}

The authors affirm that human research participants provided informed consent for publication of the article.

\section{References}

1. Plonk WM Jr, Arnold RM (2005) Terminal care: Thelast weeks of life. J Palliat Med 8:1042-1054

2. Kehl KA, andKowalkowski JA (2013) A systematic review of theprevalence of signs of impending death and symptoms in the last2 weeks of life. Am J Hosp Palliat Care 30:601-616

3. Lee IC, Kim CK, Suh SY et al (2007) Validation of scoring system for survival prediction in terminalcancer patients in Korea. J Korean Acad Fam Med 28:682-689

4. Chuang RB, Hu WY, Chiu TY et al (2004) Prediction of survival in terminal cancer patients in Taiwan: constructing a prognosticscale. J Pain Symptom Manage 28:115-122

5. Domeisen Benedetti F, Ostgathe $C$ et al (2013) International palliative care experts' view onphenomena indicating the last hours and days oflife. Support Care Cancer 21:1509-1517

6. Hwang IC, Ahn HY, Park SM et al (2012) Clinical changes in terminally cancer patients and death within 48 h: when should we refer patients to a separate room? Support Care Cancer 21:835-840

7. Hui D, Hess K, dos Santos R et al (2015) A diagnosticmodel for impending death in cancer patients. preliminary reportCancer 121:3914-3921

8. Hui D, dos Santos R, Chishoim G et al (2014) Clinical signs of impendingdeath in cancer patients, 19. Oncologist, pp 681-687 
9. Shin J, Kim SH, Suh SY et al (2020)Physical and psychological symptoms and signs in dying digestive tract cancer patients: the East Asian collaborative cross-cultural Study to Elucidate the Dying process (EASED). Support Care CancerEpub ahead of print

10. Shinjo T, Morita T, Hirai K et al (2010) Care for imminently dying cancer patients: family members' experiences and recommendations. J Clin Oncol 28:142-148

11. NICE (National Institute for Health and Care Excellence) (2015) Care of dying adults in the last days of life (NG31). NICE, London

12. Mori $\mathrm{M}$, Morita $\mathrm{T}$, Igarashi $\mathrm{N}$ et al (2018) Communication about the impending death of patients with cancer to the family: a nationwide survey. BMJ Support Palliat Care 8:221-228

13. Shimizu Y, Miyashita M, Morita T et al (2014) Care strategy for deathrattle in terminally ill cancer patients and their family members: recommendations from a cross-sectional nationwide survey of bereaved family members' perceptions. J Pain Symptom Manage 48:2-12

14. Hui D, Dos Santos R, Chisholm G et al (2015) Bedside clinical signs associated with impending death in patients with advanced cancer: preliminary findings of a prospective, longitudinal cohort study, 121. Cancer, pp 960-967

15. Hui D, dos Santos R, Chisholm G et al (2014) Clinical signs of impending death in cancer patients, 19. Oncologist, pp 681-687

16. Sakurai H, Miyashita M, Imai K et al (2019) Validation of the integrated palliative care outcome scale (IPOS)-Japanese version. Jpn J Clin Oncol 49:257-262

17. Breitbart W, Rosenfeld B, Roth A et al (1997) TheMemorial Delirium Assessment Scale. J Pain SymptomManag13:128-137

18. Oken MM, Creech RH, Tormey DC et al (1982) Toxicity and response criteria of the Eastern Cooperative Oncology Group. Am J Clin Oncol5:649-655

19. Downing GM, Wainwright W, Victoria Hospice Society (2006)Medical care of the dying, 4th ed. Victoria, BC

20. Morita T, Hyodo I, Yoshimi T et al (2005) Association between hydration volume and symptoms in terminallyill cancer patients with abdominal malignancies. Ann Oncol 16:640-647

21. Morita T, Hyodo I, Yoshimi T et al (2006) Artificial hydration therapy, laboratoryfindings, and fluid balance in terminally ill patients with abdominalmalignancies. J Pain Symptom Manag 31:130-139

22. Bruera E, Driver L, Barnes EA et al (2003) Patient-controlled methylphenidate for the management of fatigue in patients with advanced cancer: a preliminary report. $\mathrm{J}$ Clin Oncol 21:4439-4443

23. Viganò A, Dorgan M, Bruera E et al (1999) The relative accuracy of the clinical estimation of the duration of life for patients with end of life cancer. Cancer86: 170-176

24. Budhwani S, Moineddin R, Wodchis WP et al (2020) Do Longitudinally Collected Symptom Scores Predict Time to Death in Advanced Breast Cancer: A Joint Modeling Analysis. J Pain Symptom Manage59:1009-1018 
25. Efficace F, Cartoni C, Niscola P et al (2012) Predicting survival in advanced hematologic malignancies: do patient-reported symptoms matter? Eur J Haematol 89:410-416

26. Kehl KA, Kowalkowski JA (2013) A systematic review of the prevalence of signs of impending death and symptoms in the last 2 weeks of life. Am J Hosp Palliat Care 30:601-616

27. Morita T, Tsunoda J, Inoue S et al (1999) The Palliative Prognostic Index: a scoring system for survival prediction of terminally ill cancer patients. Support Care Cancer 7:128-133

28. Katz MHG, Wang H, Fleming JB et al (2009) Long-term survival after multidisciplinary management of resected pancreatic adenocarcinoma. Ann Surg Oncol 16:836-847

29. Merchant SJ, Brogly SB, Booth CM et al (2019) Palliative Care and Symptom Burden in the Last Year of Life: A Population-Based Study of Patients with Gastrointestinal Cancer. Ann Surg Oncol 26:2336-2345

30. Morita T, Tsunoda J, Inoue S et al (2002) Risk factors for death rattle in terminally ill cancer patients. A prospective exploratory study. Palliat Med 14:19-23

31. Morita T, Hyodo I, Yoshimi T et al (2004) Incidence and underlying etiologies of bronchial secretion in terminally ill cancer patients: a multicenter, prospective, observational study. J Pain Symptom Manag27:533-539

32. Bennett MI (1996) Death rattle: an audit of hyoscine (scopolamine) use and review of management.J Pain Symptom Manage12: 229-233

33. Valvani A, Martin A, Devarajan A et al (2019) Postobstructive pneumonia in lung cancer. Ann Transl Med 7:357

34. Bruera E, Schmitz B, Pither J et al (2000) The frequency and correlates of dyspnea in patients with advanced cancer. J Pain Symptom Manage 19:357-362

35. Walsh D, Donnelly S, Rybicki L (2000) The symptoms of advanced cancer: Relationship to age, gender, and performance status in 1,000 patients. Support Care Cancer 8:175-179

36. Smith EL, Hann DM, Ahles TA et al (2001) Dyspnea, anxiety, body consciousness, and quality of life in patients with lung cancer. J Pain Symptom Manage 21:323-329

37. Muers MF, Round CE (1993) Palliation of symptoms in non-small cell lung cancer: A study by the Yorkshire regional cancer organisation thoracic group, 48. Thorax, pp 339-343

38. Reuben DB, Mor V (1986) Dyspnea in terminally ill cancer patients, 89. Chest, pp 234-236

39. Heyse-Moore LH, Ross V, Mullee MA (1991) How much of a problem is dyspnoea in advanced cancer? Palliat Med5:20-26

40. Yamashita R, Arao H, Takao A et al (2017) Unfinished Business in Families of Terminally III With Cancer Patients. J Pain Symptom Manage 54:861-869

41. Yao CA, Hu WY, Lai YF et al (2007) Does dying at home influence the good death of 5 terminal cancer patients? J Pain Symptom Manage 34:497-504

42. Sakurai H, Miyashita M, Morita T et al (2021) Comparison between patient-reported and clinicianreported outcomes: Validation of the Japanese version of the Integrated Palliative care Outcome 
Scale for staff. PalliatSupport Care5:1-7

43. Pautex S, Moynier-Vantieghern K, Herrmann FR et al (2009) State of consciousness during the last days of life in patients receiving palliative care. J Pain Symptom Manage38:e1-e3

44. Hui D, dos Santos R, Chisholm G et al (2014) Clinical signs of impending death in cancer patients, 19. Oncologist, pp 681-687

\section{Tables}

Table 1. Baseline characteristics of study participants according to the type of primary cancer $(n=2131)$ 


\begin{tabular}{|c|c|c|c|}
\hline Characteristics & Digestive tract & Lung & Others \\
\hline $\mathrm{n}(\%)$ & $1042(48.9)$ & $368(17.3)$ & $721(33.8)$ \\
\hline Age (years)* & $71.3 \pm 13.0$ & $72.8 \pm 10.9$ & $70.0 \pm 14.3$ \\
\hline Survival time (days, median $[95 \% \mathrm{CI}])^{\star \star}$ & $15[2.0-71.0]$ & $15[2.0-90.2]$ & $15[2.0-79.0]$ \\
\hline \multicolumn{4}{|l|}{ Sex } \\
\hline Male & $575(55.2)$ & $239(65.0)$ & $293(40.6)$ \\
\hline Female & $467(44.8)$ & $129(35.1)$ & $428(59.4)$ \\
\hline \multicolumn{4}{|l|}{ Country } \\
\hline Japan & $777(74.6)$ & $283(76.9)$ & $571(79.2)$ \\
\hline Korea & $151(14.5)$ & $40(10.9)$ & $62(8.6)$ \\
\hline Taiwan & $114(10.9)$ & $45(12.2)$ & $88(12.2)$ \\
\hline \multicolumn{4}{|l|}{ Metastasis } \\
\hline None & $365(35.2)$ & $64(17.5)$ & $227(31.5)$ \\
\hline Liver & $499(47.9)$ & $92(25.1)$ & $221(30.7)$ \\
\hline Lung & $316(30.4)$ & $168(45.7)$ & $286(39.7)$ \\
\hline Bone & $142(13.6)$ & $164(44.7)$ & $268(37.2)$ \\
\hline Brain & $43(4.1)$ & $159(43.3)$ & $97(13.5)$ \\
\hline$\geq 2$ organs & $252(24.3)$ & $181(49.5)$ & $262(36.3)$ \\
\hline \multicolumn{4}{|l|}{ Charlson Comorbidity Index } \\
\hline 0 & $604(58.0)$ & $206(56.0)$ & $471(65.3)$ \\
\hline 1 & $258(24.8)$ & $94(25.5)$ & $156(21.6)$ \\
\hline 2 & $75(7.2)$ & 49 (13.3) & $56(7.8)$ \\
\hline$\geq 3$ & $105(10.1)$ & $19(5.2$ & $38(5.3)$ \\
\hline \multicolumn{4}{|l|}{ Highest education level } \\
\hline Illiterate/Elementary/Junior high & $166(15.9)$ & $47(12.8)$ & $79(11.0)$ \\
\hline High school/Some college & $158(15.2)$ & $53(14.4)$ & $112(15.5)$ \\
\hline College/Graduate school & $83(8.0)$ & $30(8.2)$ & $72(10.0)$ \\
\hline Unknown & $635(60.9)$ & $238(64.7)$ & $458(63.5)$ \\
\hline
\end{tabular}




\begin{tabular}{|llll|} 
Unmarried & $89(8.5)$ & $27(7.3)$ & $85(11.8)$ \\
Widowried & $652(62.6)$ & $247(67.1)$ & $425(59.0)$ \\
Unknown & $301(28.9)$ & $93(25.3)$ & $210(29.1)$ \\
ECOG PS & 0 & $1(0.3)$ & $1(0.1)$ \\
$0-1$ & & & \\
2 & $16(1.5)$ & $3(0.8)$ & $8(1.1)$ \\
3 & $87(8.4)$ & $24(6.5)$ & $45(6.2)$ \\
4 & $428(41.1)$ & $146(39.7)$ & $277(38.4)$ \\
\hline KPS* & $511(49.0)$ & $195(53.0)$ & $391(54.2)$ \\
$10-40$ & & & \\
$50-70$ & $752(72.2)$ & $285(77.5)$ & $549(76.3)$ \\
$80-90$ & $280(26.9)$ & $81(22.0)$ & $168(23.3)$ \\
\hline
\end{tabular}

Data are presented as mean \pm standard deviation or number (percentage).

Abbreviations: Cl, confidence interval; ECOG PS, Eastern Cooperative Oncology Group performance status; KPS, Karnofsky Performance Scale

Footnote: Digestive tract cancer includes gastroesophageal, colorectal, hepatobiliary, and pancreatic cancers. Lung cancer includes small-cell and non-small cell types. Others include breast, gynecological, urological, head and neck, bone and soft tissue, skin, brain and hematological malignancies.

*missing value $(\mathrm{n}=1)$

$\star \star$ missing value $(\mathrm{n}=2)$

Table 2. Prevalence of symptoms and signs according to the type of primary cancer in patients in the last 3 days of life $(n=2131)$ 


\begin{tabular}{|c|c|c|c|c|}
\hline Symptoms and signs & Digestive tract & Lung & Others & Total \\
\hline \multicolumn{5}{|l|}{ Fatigue } \\
\hline Present & $637(61.1)$ & $190(51.6)$ & $386(53.5)$ & $1213(56.9)$ \\
\hline Absent & $54(5.2)$ & $35(9.5)$ & $44(6.1)$ & $133(6.2)$ \\
\hline Unconscious & $351(33.7)$ & $143(38.9)$ & $291(40.4)$ & $785(36.8)$ \\
\hline \multicolumn{5}{|l|}{ Dry mouth } \\
\hline Present & $549(52.7)$ & $176(47.8)$ & $318(44.1)$ & $1043(48.9)$ \\
\hline Absent & $153(14.7)$ & $55(15.0)$ & $116(16.1)$ & $324(15.2)$ \\
\hline Unconscious & $340(32.6)$ & $137(37.2)$ & $287(39.8)$ & $764(35.8)$ \\
\hline \multicolumn{5}{|l|}{ Drowsiness } \\
\hline Present & $559(53.7)$ & $185(50.3)$ & $339(47.0)$ & $1083(50.8)$ \\
\hline Absent & $129(12.4)$ & $44(12.0)$ & $92(12.8)$ & $265(12.4)$ \\
\hline Unconscious & $354(34.0)$ & $139(37.8)$ & $290(40.2)$ & $783(36.7)$ \\
\hline \multicolumn{5}{|l|}{ Dyspnea } \\
\hline Present & $363(34.8)$ & $206(56.0)$ & $308(42.7)$ & $877(41.1)$ \\
\hline Absent & $633(60.8)$ & $143(38.9)$ & $393(54.5)$ & $1169(54.9)$ \\
\hline Unconscious & $46(4.4)$ & $19(5.2)$ & $20(2.8)$ & $85(4.0)$ \\
\hline \multicolumn{5}{|l|}{ Delirium* } \\
\hline Present & $292(28.1)$ & $107(29.1)$ & $186(25.8)$ & $585(27.4)$ \\
\hline Absent & $749(72.0)$ & $261(70.9)$ & $535(74.2)$ & $1545(72.5)$ \\
\hline \multicolumn{5}{|l|}{ Hallucination } \\
\hline Present & $316(30.3)$ & $106(28.8)$ & $191(26.5)$ & $613(28.8)$ \\
\hline Absent & $726(69.7)$ & $262(71.2)$ & $530(73.5)$ & $1518(71.2)$ \\
\hline \multicolumn{5}{|l|}{ Respiratory secretion* } \\
\hline Present & $401(38.5)$ & $206(56.1)$ & $321(44.5)$ & $928(43.6)$ \\
\hline Absent & $641(61.5)$ & $161(43.9)$ & $400(55.5)$ & $1202(56.4)$ \\
\hline \multicolumn{5}{|c|}{ Edema of lower extremities** } \\
\hline Present & $709(68.1)$ & $170(46.2)$ & $451(62.6)$ & $1330(62.4)$ \\
\hline Absent & $332(31.9)$ & $198(53.8)$ & $269(37.4)$ & $799(37.5)$ \\
\hline
\end{tabular}




\begin{tabular}{|lllll|}
\hline Ascites* & & & & \\
Present & $479(46.0)$ & $20(5.4)$ & $157(21.8)$ & $656(30.8)$ \\
Absent & $562(54.0)$ & $348(94.6)$ & $564(78.2)$ & $1474(69.2)$ \\
Myoclonus & & & & \\
Present & $75(7.2)$ & $23(6.3)$ & $58(8.0)$ & $156(7.3)$ \\
Absent & $967(92.8)$ & $345(93.8)$ & $663(92.0)$ & $1975(92.7)$ \\
\hline
\end{tabular}

*missing value $(n=1)$

$\star \star$ missing value $(\mathrm{n}=2)$

Data are presented as numbers (percentages).

Footnote: Digestive tract cancer includes gastroesophageal, colorectal, hepatobiliary, and pancreas cancers. Lung cancer includes small-cell and non-small cell types. Others includes breast, gynecological, urological, head and neck, bone and soft tissue, skin, brain and hematological malignancies.

Table 3. The severity of symptoms and signs in the last 3 days of life according to the type of primary cancer $(n=2131)$

\begin{tabular}{|c|c|c|c|c|c|}
\hline Symptoms and signs & Range & Digestive tract & Lung & Others & $p$ value \\
\hline Fatigue* & $0-4$ & $2.19 \pm 1.18^{a}$ & $1.96 \pm 1.26^{\mathrm{b}}$ & $2.01 \pm 1.19^{b}$ & 0.01 \\
\hline Dry mouth* & $0-4$ & $1.47 \pm 1.15^{\mathrm{a}}$ & $1.37 \pm 1.09 a b$ & $1.29 \pm 1.09^{b}$ & 0.03 \\
\hline Drowsiness* & $0-4$ & $1.71 \pm 1.25$ & $1.70 \pm 1.22$ & $1.56 \pm 1.20$ & 0.12 \\
\hline Dyspnea* & $0-2$ & $0.60 \pm 0.85^{a}$ & $1.05 \pm 0.93^{b}$ & $0.78 \pm 0.93^{c}$ & $<0.01$ \\
\hline Delirium & $0-3$ & $0.40 \pm 0.72$ & $0.45 \pm 0.79$ & $0.39 \pm 0.73$ & 0.42 \\
\hline Hallucination & $0-3$ & $0.49 \pm 0.86$ & $0.46 \pm 0.84$ & $0.42 \pm 0.81$ & 0.24 \\
\hline Respiratory secretion & $0-3$ & $0.58 \pm 0.84^{a}$ & $0.88 \pm 0.95^{b}$ & $0.70 \pm 0.90^{c}$ & $<0.01$ \\
\hline Edema of lower extremities & $0-4$ & $1.19 \pm 1.05^{\mathrm{ac}}$ & $0.71 \pm 0.93^{b}$ & $1.14 \pm 1.09^{\mathrm{c}}$ & $<0.01$ \\
\hline Ascites & $0-2$ & $0.68 \pm 0.81^{a}$ & $0.08 \pm 0.37^{b}$ & $0.30 \pm 0.61^{c}$ & $<0.01$ \\
\hline Myoclonus & $0-4$ & $0.10 \pm 0.44$ & $0.10 \pm 0.45$ & $0.13 \pm 0.50$ & 0.51 \\
\hline
\end{tabular}

*Unconscious patients are included under missing data 
Data are presented as mean \pm standard deviation.

$P$ values are based on ANOVA.

Different superscripts indicate statistically different groups after post-hoc analysis using Turkey-Kramer test.

Footnote: Digestive tract cancer includes gastroesophageal, colorectal, hepatobiliary, and pancreatic cancers. Lung cancer includes small-cell and non-small cell types. Others includes breast, gynecological, urological, head and neck, bone and soft tissue, skin, brain and hematological malignancies.

\section{Figures}

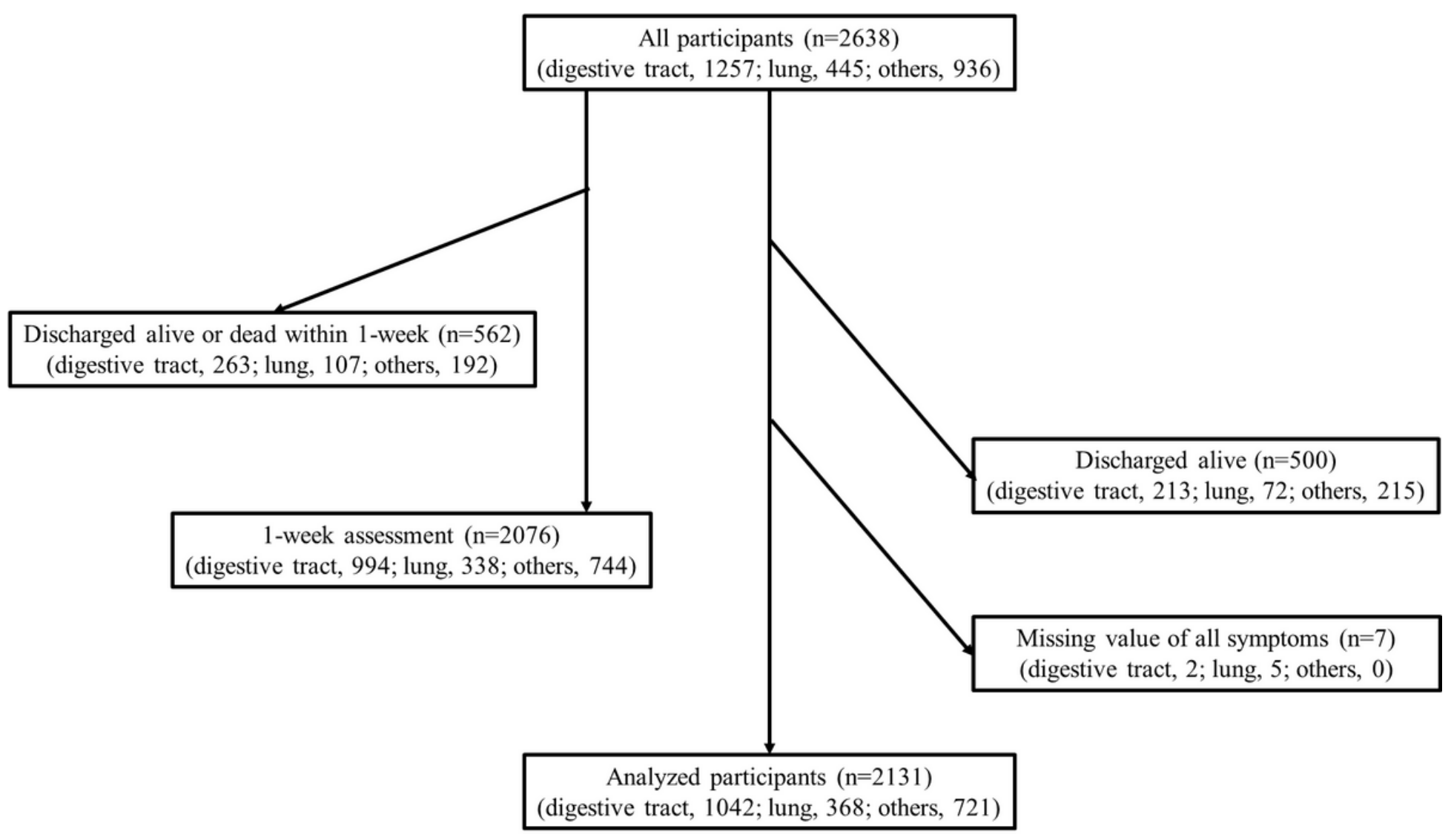

\section{Figure 1}

Flow chart outlining participant selection 


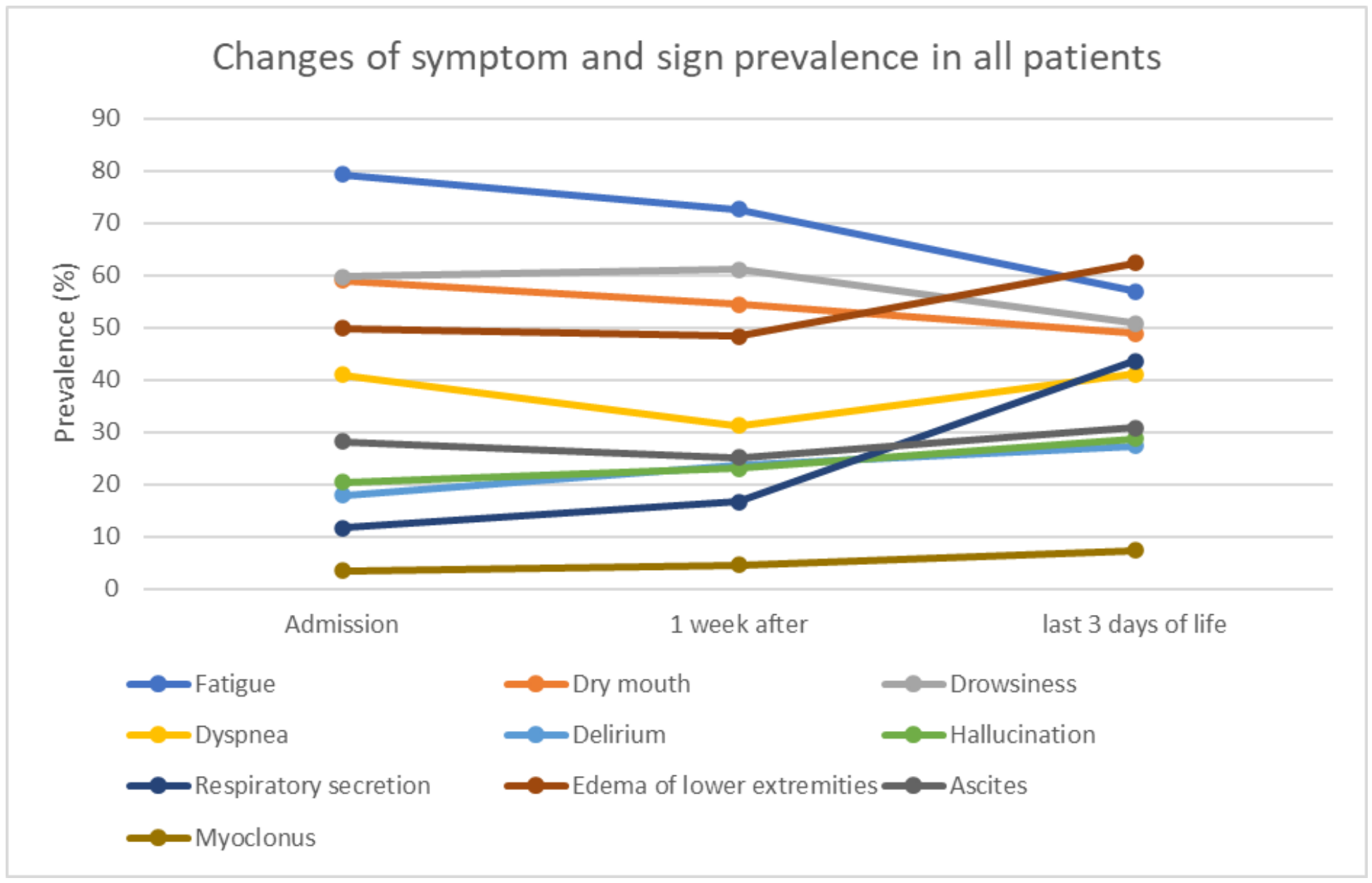

Figure 2

Changes in the prevalence of symptoms and signs in all patients $(n=2076)$

\section{Supplementary Files}

This is a list of supplementary files associated with this preprint. Click to download.

- STROBEchecklist.docx 